REVISTA INTERNACIONAL DE CIENCIAS DEL DEPORTE International Journal of Sport Science

Rev. int. cienc. deporte

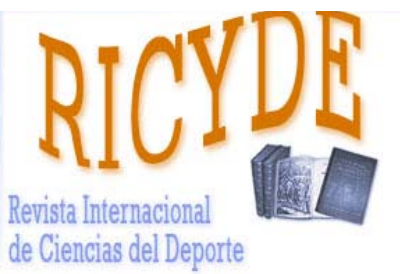

International Journal of Sport Science VOLUMEN VIII - AÑO VIII

Páginas:258-271 ISSN:1885-3137 No 29 - Julio - 2012

\title{
Diseño y aplicación de un programa de intervención de práctica deportiva inclusiva y su efecto sobre la actitud hacia la discapacidad: El Campus Inclusivo de Baloncesto.
}

\section{Design and implementation of an intervention program of inclusive sport and its effect on attitudes toward disability: The Inclusive Basketball Campus}

\author{
Javier Pérez-Tejero, Carmen Ocete-Calvo \\ Universidad Politécnica de Madrid. España \\ Gema Ortega-Vila \\ Fundación Real Madrid \\ Javier Coterón-López \\ Universidad Politécnica de Madrid. España
}

\section{Resumen}

El objetivo del presente estudio fue diseñar y aplicar un programa de intervención como modelo de práctica deportiva inclusiva, analizando el impacto que ejerce en la actitud hacia la discapacidad en jugadores que no la tienen. Para ello, se puso en práctica el "Campus Inclusivo de Baloncesto", organizado por la Fundación Real Madrid, como actividad deportiva inclusiva donde la práctica del baloncesto sirve de contexto para una práctica normalizadora, ya que a él asisten participantes con y sin discapacidad física. El diseño del estudio fue de tipo experimental, utilizando el "Cuestionario de actitudes hacia las personas con discapacidad" (Reina, López, Jiménez, García-Calvo, y Hutzler, 2011), aplicándolo antes de la intervención, después y pasados 9 meses de la misma, a los 21 participantes sin discapacidad (17 hombres y 4 mujeres), con edades comprendidas entre los 8 y los 14 años. Se aplicaron la prueba de Wilcoxon y ANOVA de medidas repetidas, estableciendo el nivel de confianza en $p \leq 0,05$. Los resultados muestran cambios significativos en positivo sobre la actitud hacia las personas con discapacidad tras el desarrollo de la actividad y un mantenimiento de dichos cambios en el tiempo. Estos resultados sugieren la validez del diseño propuesto para promover cambios en la perspectiva de la inclusión en contextos de iniciación deportiva.

Palabras clave: baloncesto; inclusión; discapacidad física; actitudes; cuestionario.

\section{Abstract}

The aim of this study was to design and to implement an intervention program as a model of inclusive sport, analyzing its impact on the attitude towards disability in children who have no disability. For this purpose, the Inclusive Basketball Campus was implemented, organized by the Real Madrid Foundation, where basketball as inclusive sport provides a context for normalizing practice, since this activity is attended by participants with and without physical disabilities. The study design was experimental, using the "Survey of attitudes towards people with disabilities" (Reina, López, Jiménez, García-Calvo \& Hutzler, 2011) applying it before intervention, just after intervention and 9 months later on a sample of 21 participants without disabilities (17 males and 4 females) aged between 8 and 14 . Wilcoxon and ANOVA's repeated measures tests were applied, setting the confidence level at $p \leq 0.05$. The results show that, after the activity, there are significant changes in positive on the attitude towards people with disabilities, and these changes keep in time. These results suggest the validity of the proposed design to promote changes on the inclusion perspective in the context of sports initiation.

Key words: basketball; inclusion; physical disability; attitudes; questionnaire.

Correspondencia/correspondence: : Javier Pérez Tejero

Centro de Estudios sobre Deporte Inclusivo. Facultad de Ciencias de la Actividad Física y del DeporteINEF. Universidad Politécnica de Madrid. España

E-mail: j.perez@upm.es 


\section{Introducción}

$\mathrm{L}$ a Unión Europea se refiere a la inclusión social como aquel proceso asegurador de que personas en riesgo de pobreza y exclusión social aumenten las oportunidades y los recursos necesarios para participar activamente en la vida económica, social y cultural, y de que a su vez, gocen de unas condiciones de vida y de bienestar que se consideran normales en la sociedad en la que viven (Ríos, y col., 2009). Un papel fundamental y determinante en la inclusión de chicos con discapacidad en contextos generales lo desempeñan las actitudes (Conaster, Block, y Gansneder, 2002; FolsomMeeky Rizzo, 2002; Hodge, Murata, y Kozub, 2002), siendo consideradas las actitudes favorables un factor clave para obtener una inclusión exitosa (Heikinaro-Johansson, French, Sherrill, y Huuhka, 1995; Hodge, Davis, Woodard, y Sherrill, 2002). En el ámbito educativo y según lo expuesto, una de las grandes barreras para la inclusión educativa de chicos con discapacidad en las clases regulares es la actitud de los compañeros hacia éstos (Xafopoulos, Kudlacek, y Evaggelinou, 2009). Sherrill (2003) distingue dos tipos de inclusión: desde el nivel físico en la que alumnos con y sin discapacidad comparten un mismo espacio, y a nivel social, en la que la interacción entre personas con y sin discapacidad se produce a nivel de hogar, escuela y comunidad.

Se debe prestar especial atención al papel fundamental que desempeñan las actitudes en la inclusión de personas con discapacidad ya que, en función de cómo sean éstas, encontraremos verdaderos obstáculos o una verdadera intención de conseguir la inclusión. Sherrill (1998, p. 7), autora para la que la clave del cambio de los comportamientos en las personas está en la actitud, la define como "una serie de creencias cargadas de emoción que predisponen a la persona a ciertos tipos de comportamientos". Para Ruiz (2002), las actitudes poseen tres componentes: a) cognitivo: las ideas o creencias que tenemos, b) afectivo: las emociones que tenemos asociadas a esas ideas, c) conductual: cómo nos comportamos según los componentes anteriores. Existen una serie de postulados sobre las actitudes que se debe de considerar cuando se trabaja sobre ellas: son aprendidas mediante la interacción con otras personas; son complejas, ya que poseen componentes múltiples; son relativamente estables; tienen un objeto social específico como referente; pueden cambiar en cantidad y calidad, es decir en intensidad o dirección; y son manifestaciones del comportamiento (Antonak y Livneh, 1988).

Según la teoría de acción razonada (Ajzen y Fishbein, 1980), posteriormente denominada por Azjen teoría del comportamiento o la conducta planeada (theory of planned behaviour), las actitudes vienen determinadas por las creencias que interactúan influyendo en nuestras intenciones, lo que determinará posteriormente nuestro comportamiento. Estas creencias son producto de nuestras experiencias pasadas, nuestro conocimiento y la nueva información que adquirimos (Kowalski y Rizzo, 1996), influyendo sobre las actitudes que manifestamos. Si las experiencias y creencias de la sociedad se manifiestan en actitudes negativas hacia las personas con discapacidad, la consecuencia última es que éstas interiorizan esas actitudes construyendo una percepción negativa de sí mismos (Finkelsteins y French, 1993). Estas autopercepciones son dinámicas, participando activamente la persona con discapacidad en su construcción, constituyéndolas, confirmándolas y transformándolas a través de las interacciones con los demás (Williams, 1994). 
Pérez-Tejero, J.; Ocete-Calvo, C.; Ortega-Vila, G.; Coterón-López, J. (2012). Diseño y aplicación de un programa de intervención de práctica deportiva inclusiva y su efecto sobre la actitud hacia la

discapacidad: El Campus Inclusivo de Baloncesto. RICYDE. Rev. int. cienc. deporte. 29(8), 258-271. http://dx.doi.org/10.5232/ricyde2012.02905

Las actitudes hacia las personas con discapacidad pueden ser mejoradas mediante, al menos, cuatro estrategias: la información, el contacto directo (CD), la persuasión y la experiencia vicaria (Shaver, 1987), en la que el individuo, observando a otras personas ejecutar exitosamente ciertas actividades, puede llegar a creer que él mismo posee las capacidades suficientes para desempeñarlas con igual éxito. Las estrategias que se han mostrado más eficaces para promover el cambio de actitudes hacia la discapacidad son la información y el CD (Flórez, Aguado, y Alcedo, 2009; Slininger, Sherrill, y Jankowski, 2000). Cuando a las personas implicadas en un programa de practica físicodeportiva se les facilita información acerca de la discapacidad, los deportes adaptados y su práctica, muestran una actitud más positiva hacia la inclusión de personas con discapacidad a través del deporte (Krahé y Altwasser, 2006; Liu, Kudlacek y Ondrej, 2010). A su vez, cuando se produce un contacto entre personas con y sin discapacidad en una situación de práctica deportiva cooperativa, ésta reporta beneficios mutuos que pueden conllevar a una mejora de las actitudes (Tripp, French, y Sherrill, 1995; Wilhite, Mushett, Goldenberg, y Trader, 1997). Este CD en el ámbito de la actividad física, se puede dar en tres contextos: el educativo, el deportivo y el recreativo/social (Reina, 2003). Nos centraremos en este último por ser el ámbito en el que desarrollamos la intervención objeto de estudio, ya que la integración de personas con discapacidad en este ámbito es esencial para poder completar el proceso de normalización en el que son valoradas de forma individual (Brasile, 1990), influyendo su participación en actividades recreativas sobre cuatro aspectos de la autopercepción física: proporciona nuevas experiencias corporales, realza la percepción de sus logros físicos, redefine sus capacidades físicas e incrementa su confianza para la realización de nuevas actividades físicas (Blinde y McClung, 1997).

La teoría del contacto (Allport, 1954) explica cómo se forman las actitudes, estableciendo que la discriminación y los prejuicios hacia un grupo minoritario pueden reducirse por el CD entre individuos, produciendo cambios en las actitudes. Se sustenta en 4 postulados fundamentales: igualdad de estatus dentro del grupo, objetivos comunes, cooperación entre individuos y apoyo a nivel institucional y legislativo. Además, considera que las intervenciones diseñadas para provocar el cambio en las actitudes a través del CD han de tener en cuenta: a) los aspectos cuantitativos del contacto: frecuencia, duración, número de personas; b) el estado del contacto: inferioridad, igualdad, superioridad; c) rol dentro del contacto: competitivo o cooperativo; d) contexto social: real o artificial o voluntario $u$ obligatorio; e) experiencias individuales personales: contacto previo con personas con discapacidad, por ejemplo, y f) ámbito del contacto: educativo, recreativo, etc. Este contacto también debe ser estructurado, ya que el producido de manera casual no es efectivo (Archie y Sherrill, 1989). Además debe caracterizarse por promover el logro de objetivos comunes y el respeto de las diferencias individuales, ha de ser realizado con frecuencia, han de ser gratificantes y placenteros, deben tener carácter significativo y ha de realizarse un seguimiento y control sobre la interacción producida (Sherrill, 1998).

Se han encontrado escasos estudios en la literatura cientifica revisada que hayan utilizado el CD. Roper (1990), en un estudio plenteado en el ámbito educativo, comprobó la importancia de incluir el CD para mejorar las actitudes. Slininger y col. (2000) constataron la importancia de estructurar el CD para obtener efectos positivos. Coincidiendo con estos últimos, Tripp y col. (1995), obtuvieron resultados positivos en el cambio de actitud hacia la discapacidad respecto a las personas con trastornos en el comportamiento, pero no hacia la discapacidad física. Reforzando lo anterior, Bergman 
y Handson (2000) en un campus recreativo de actividades deportivas, no obtuvieron resultados positivos tras el CD entre personas con y sin discapacidad, pudiendo deberse a que el CD no fue estructurado, produciéndose éste de manera casual. Por otro lado, Gillespie (2002) en otro campus recreativo de baloncesto, utilizó la simulación de la discapacidad en silla de ruedas como técnica de cambio de actitudes, encontrando modificaciones significativas en la actitud de los participantes.

En la actualidad no existen en la literatura de nuestro país estudios sobre los beneficios de una práctica deportiva inclusiva entre chicos con y sin discapacidad a nivel recreativo o de iniciación deportiva. Por este motivo es necesario proporcionar modelos de intervención que faciliten la integración social, proceso complejo en el cual intervienen diferentes agentes y variables, y cuyas actitudes pueden determinar la inclusión de forma decisiva (Siperstein, Norins, Corbin, y Shirver, 2003). Por todo lo anterior, el presente trabajo tiene dos objetivos; por un lado, diseñar una propuesta de intervención como modelo de práctica deportiva inclusiva y, por otro lado, analizar el impacto que ejerce dicha propuesta de intervención sobre la actitud hacia la discapacidad en los participantes que no la tienen, evaluando su permanencia en el tiempo.

\section{Método}

\section{Muestra}

La muestra del estudio está compuesta por 21 sujetos sin discapacidad (17 varones y 4 mujeres) como grupo experimental, con edades comprendidas entre los 8 y los 14 años, con una media de $11,4( \pm 0,6)$ años. Los participantes practican baloncesto durante dos horas a la semana en las escuelas de la Fundación del Real Madrid distribuidas por la capital madrileña, aparte de la Educación Física escolar.

\section{Diseño}

El modelo de intervención propuesto se llevó a cabo durante la II edición del "Campus Inclusivo de Baloncesto", en Junio de 2010. Este campus está organizado por la Fundación Real Madrid con la colaboración del Centro de Estudios sobre Deporte Inclusivo (INEF-UPM) y la Fundación Sanitas. Los participantes convivieron durante cuatro días completos en las instalaciones de la "Ciudad del Fútbol" en las Rozas (Madrid), formando parte de un único grupo (Pérez, Ortega, Trigo, y Pinilla, 2009). Los objetivos plateados en el Campus fueron: a) ofrecer un contexto de práctica inclusivo en el que los participantes, con interés por el baloncesto, encuentren un lugar para su disfrute, tanto en su versión a pie como en silla de ruedas; b) facilitar el aprendizaje táctico-técnico del baloncesto, valorando la diversidad y capacidad que presenta cada jugador, ofreciendo situaciones de práctica conjunta a todos los niveles, y c) realizar las actividades en un entorno lúdico y accesible que promueva el disfrute de los jugadores, transmitiendo los valores positivos del deporte en un marco de convivencia que favorezca las relaciones interpersonales.

Tomando los postulados de la teoría del contacto como referencia y las aportaciones de la literatura científica anteriormente citadas, se diseñó una propuesta de intervención que recogiese, de forma estructurada, el desarrollo de una actividad de carácter inclusivo conforme a lo que la investigación de los últimos años viene proponiendo, con el fin de mejorar la calidad y profundidad de los resultados en el cambio de actitudes. 
Pérez-Tejero, J.; Ocete-Calvo, C.; Ortega-Vila, G.; Coterón-López, J. (2012). Diseño y aplicación de un programa de intervención de práctica deportiva inclusiva y su efecto sobre la actitud hacia la discapacidad: El Campus Inclusivo de Baloncesto. RICYDE. Rev. int. cienc. deporte. 29(8), 258-271. http://dx.doi.org/10.5232/ricyde2012.02905

En las Tablas 1 y 2 se recogen los fundamentos teóricos en los que nos apoyamos y las actuaciones diseñadas para llevarlos a cabo en la práctica.

Tabla 1. Características de la intervención basadas en los postulados y variables de la Teoría del Contacto (Allport, 1954).

\begin{tabular}{|c|c|c|}
\hline & & PROPUESTA DE INTERVENCIÓN \\
\hline \multirow{4}{*}{ POSTULADOS } & Igualdad de estatus & $\begin{array}{l}\text { Distribución de los grupos y asignación del material adaptado por } \\
\text { parte del técnico, facilitando los apoyos y recursos necesarios para } \\
\text { acceder a la práctica. }\end{array}$ \\
\hline & Objetivos comunes & Tareas donde todos deben participan para conseguir un objetivo. \\
\hline & Cooperación entre individuos & $\begin{array}{l}\text { Situaciones inclusivas a través fundamentalmente de actividades } \\
\text { cooperativas. }\end{array}$ \\
\hline & Apoyo institucional & $\begin{array}{l}\text { Fundación Real Madrid, Fundación Sanitas, Centro Estudios sobre } \\
\text { Deporte Inclusivo, INEF-Universidad Politéenica de Madrid. }\end{array}$ \\
\hline \multirow{6}{*}{ VARIABLES } & Aspectos cuantitativos & $\begin{array}{l}\text { Grupo reducido: } 21 \text { chicos sin discapacidad y } 7 \text { con discapacidad } \\
\text { física. Duración: } 4 \text { días de actividad. }\end{array}$ \\
\hline & Estado del contacto & $\begin{array}{l}\text { Igualdad de condiciones, compartiendo espacios y tiempos de } \\
\text { práctica y libre. } 3 \text { sesiones de entrenamiento diarios: } \\
\text { Sesión 1. Espacios separados/Objetivos específicos de cada } \\
\text { modalidad/ } 1,5 \text { horas. } \\
\text { Sesión 2. Espacio común/Aplicación de fundamentos } \\
\text { aprendidos en la sesión anterior/ } 1 \mathrm{~h} \text {. } \\
\text { Sesión 3. Espacios inicialmente por separado para terminar de } \\
\text { forma conjunta/ Actividades inclusivas/2h. }\end{array}$ \\
\hline & Rol dentro del contacto & Cooperación. \\
\hline & Contexto social & Participación voluntaria. \\
\hline & Experiencia personal & No contacto previo con personas con discapacidad. \\
\hline & Ámbito del contacto & $\begin{array}{l}\text { Ocio y recreación. Técnicos con experiencia previa en el deporte } \\
\text { inclusivo con cualificación específica en baloncesto, ciencias del } \\
\text { deporte e inclusión educativa. }\end{array}$ \\
\hline
\end{tabular}

Tabla 2. Características de la intervención según aportaciones de diferentes autores.

\begin{tabular}{|c|c|l|}
\hline \multirow{2}{*}{ AUTORES } & CONSIDERACIONES & \multicolumn{1}{|c|}{ PROPUESTA DE INTERVENCIÓN } \\
\hline Archie y Sherrill, (1989) & $\begin{array}{c}\text { Estructurado: Control de } \\
\text { interacción entre chicos con y } \\
\text { sin discapacidad }\end{array}$ & $\begin{array}{l}\text { Planificación previa: sesiones y entrenamientos, actividades } \\
\text { lúdicas y veladas, reunión con familiares y evaluación al finalizar } \\
\text { el día. Previsión día posterior. }\end{array}$ \\
\cline { 2 - 3 } Sherrill, (1998) & Gratificante & CD a través de la práctica del deporte elegido. \\
\cline { 2 - 3 } & Frecuente & $\begin{array}{l}\text { Cuatro días de convivencia durante entrenamientos, tiempo libre y } \\
\text { residencia. Grupo único: calentamiento, vuelta a la calma y puesta } \\
\text { en común. }\end{array}$ \\
\hline $\begin{array}{c}\text { (Tripp, y col., 1995; } \\
\text { Wilhite, y col., 1997) }\end{array}$ & $\begin{array}{l}\text { Centrándonos en las habilidades requeridas para la práctica } \\
\text { indicalica deportiva en lugar de la discapacidad. }\end{array}$ \\
\hline
\end{tabular}

La estructura de un día cualquiera del campus consistió en tres sesiones de entrenamiento diferenciadas. La primera posee unos objetivos específicos para cada modalidad, a pie y en silla (10.00-11.30 horas); la segunda favorece la inclusión de ambos grupos aplicando los fundamentos aprendidos en cada modalidad a la actividad conjunta (12.00-13.00 horas); y en la tercera sesión de entrenamiento los grupos 
practican de manera conjunta mediante actividades inclusivas (17.00-19.00 horas). Para todas las sesiones, las situaciones de calentamiento y vuelta a la calma fueron conjuntas.

El cuestionario fue administrado por los técnicos deportivos del propio campus. El tiempo necesario para su cumplimentación fue de 20 minutos el primer día de campus antes del comienzo de las actividades (momento 1). Tras la intervención, se administró de nuevo el último día, al finalizar el campus (momento 2). Con el fin de comprobar si los cambios producidos tras la intervención se mantenían en el tiempo, a los 9 meses se contactó con los padres para administrar de nuevo el cuestionario a sus hijos, participantes en la actividad (momento 3). En este caso, se envió por correo electrónico, devolviéndolo por el mismo medio. Para este tercer momento, 8 sujetos finalmente no contestaron, por lo que la muestra final fue de 13 participantes (11 varones y 2 mujeres).

\section{Instrumento}

Para evaluar el impacto de la práctica deportiva inclusiva sobre la actitud hacia la discapacidad de los participantes, se administró el "Cuestionario de actitudes hacia las personas con discapacidad" (Reina, Jiménez, López, y Menayo, 2008), versión modificada del "Attitudes Toward Disability Questionnaire" (ATDQ; López Ramos, 2005), adaptación de la versión original de Verdugo, Jenaro, y Arias (1994). Está compuesto por 17 ítems sobre la actitud en situaciones concretas hacia personas con discapacidad que evalúa las ideas personales (9 ítems) y la predisposición para la acción (8 ítems) respecto de este colectivo, siendo redactados como afirmaciones negativas hacia la inclusión. Utiliza una escala de tipo Likert de 5 posibilidades ( $1=$ muy desacuerdo a $5=$ muy de acuerdo).

\section{Análisis estadístico}

Se aplicaron las pruebas de Kolmogorov-Smirnof, asimetría y curtosis, observando la media, mediana, moda y los histogramas de normalidad, todo ello para comprobar la normalidad de los datos y determinar el tipo de estadística a utilizar. Los datos no cumplían los criterios de normalidad, y por ello se aplicaron pruebas estadísticas no paramétricas. Después de un análisis descriptivo de los datos (media, desviación tipica, mínimo y máximo) y para evaluar las diferencias entre los momentos 1,2 y 3 del estudio, se aplicó la prueba de Wilcoxon. Para comprobar si los cambios producidos en las actitudes de los participantes se mantenían en el tiempo, se utilizó ANOVA de medidas repetidas, haciendo un ajuste para comparaciones múltiples de Bonferroni para los momentos 2 y 3 , exclusivamente. Debido a la mortalidad de 8 sujetos de la muestra en el momento 3, se realizó el análisis con una muestra de 13 sujetos para ambos momentos. Los datos fueron tratados con el paquete estadístico SPSS para Windows, v.19.0 (Chicago, IL). Se consideró significación estadística el valor de $\mathrm{p} \leq 0,05$.

\section{Resultados}

Para el análisis descriptivo de los resultados "del cuestionario en los momentos 1 y 2 ", confirmamos que entre la medida pretratamiento y la postratamiento la diferencia entre las medias era favorable a la segunda, encontrando así una mejora en la actitud hacia las personas con discapacidad. Para todos los ítems evaluados (momentos 1 y 2) existe una disminución de la media, excepto en el ítem 13 (ver tabla 3). Los ítems en los que encontramos diferencias significativas son: 1, 3, 5 y 15 (ideas personales) y el 13 (predisposición a la acción). 
Pérez-Tejero, J.; Ocete-Calvo, C.; Ortega-Vila, G.; Coterón-López, J. (2012). Diseño y aplicación de un programa de intervención de práctica deportiva inclusiva y su efecto sobre la actitud hacia la

discapacidad: El Campus Inclusivo de Baloncesto. RICYDE. Rev. int. cienc. deporte. 29(8), 258-271. http://dx.doi.org/10.5232/ricyde2012.02905

Tabla 3. Resultados descriptivos del "Cuestionario de actitudes hacia las personas con discapacidad" (Reina, y col., 2008) para los momentos 1 y 2.

\begin{tabular}{|c|c|c|c|c|c|c|c|c|c|}
\hline ÍTEM & Momento & $\mathbf{N}$ & $\mathbf{X}$ & DE & ÍTEM & Momento & $\mathbf{N}$ & $\mathbf{X}$ & DE \\
\hline \multirow{2}{*}{1} & 1 & 20 & 3,45 & 1,50 & \multirow{2}{*}{10} & 1 & 20 & 3,60 & 1,39 \\
\hline & 2 & 20 & 2,30 & 1,34 & & 2 & 20 & 3,15 & 1,63 \\
\hline \multirow{2}{*}{2} & 1 & 21 & 1,33 & ,96 & \multirow{2}{*}{11} & 1 & 20 & 1,40 & 1,04 \\
\hline & 2 & 21 & 1,24 &, 88 & & 2 & 20 & 1,00 &, 00 \\
\hline \multirow{2}{*}{3} & 1 & 20 & 3,60 & 1,27 & \multirow{2}{*}{12} & 1 & 21 & 1,71 & 1,10 \\
\hline & 2 & 20 & 2,75 & 1,44 & & 2 & 21 & 1,43 &, 87 \\
\hline \multirow{2}{*}{4} & 1 & 21 & 1,38 &, 59 & \multirow{2}{*}{13} & 1 & 21 & 1,05 & ,21 \\
\hline & 2 & 21 & 1,24 & ,62 & & 2 & 21 & 1,76 & 1,13 \\
\hline \multirow{2}{*}{5} & 1 & 20 & 3,00 & 1,48 & \multirow{2}{*}{14} & 1 & 20 & 2,00 & 1,17 \\
\hline & 2 & 20 & 2,30 & 1,26 & & 2 & 20 & 1,80 & 1,15 \\
\hline \multirow{2}{*}{6} & 1 & 19 & 1,58 & 1,21 & \multirow{2}{*}{15} & 1 & 21 & 3,33 & 1,52 \\
\hline & 2 & 19 & 1,05 & ,22 & & 2 & 21 & 2,33 & 1,59 \\
\hline \multirow{2}{*}{7} & 1 & 21 & 1,90 & 1,41 & \multirow{2}{*}{16} & 1 & 21 & 2,14 & 1,45 \\
\hline & 2 & 21 & 1,67 & 1,01 & & 2 & 21 & 2,05 & 1,20 \\
\hline \multirow{2}{*}{8} & 1 & 21 & 3,57 & 1,39 & \multirow{2}{*}{17} & 1 & 21 & 1,62 & 1,11 \\
\hline & 2 & 21 & 2,95 & 1,43 & & 2 & 21 & 1,24 &, 70 \\
\hline \multirow{2}{*}{9} & 1 & 20 & 1,45 & 1,05 & & & & & \\
\hline & 2 & 20 & 1,05 & ,22 & & & & & \\
\hline
\end{tabular}

El item 1 ("Pienso que las personas con discapacidad tienen mayores dificultades que el resto de personas para conseguir los mismos logros personales y/o profesionales") obtiene una $\mathrm{p}=0,003$, siendo el ítem que más diferencia presenta entre el momento $1 \mathrm{y}$ 2. El ítem 5, ("Las personas con discapacidad no pueden realizar la mayoría de los deportes practicados por personas sin discapacidad"), el 13 ("No participaría en competiciones deportivas conjuntamente con personas con discapacidad") y el 15 ("Los deportes para personas con discapacidad deben tener una estructura sencilla, con pocas reglas") obtienen una significación de $\mathrm{p}=0,013, \mathrm{p}=0,017 \mathrm{y} \mathrm{p}=0,016$ respectivamente. El ítem 3 ("Los ciegos siempre han de llevar un guía que los acompañe de un lado para otro") posee una significación de $\mathrm{p}=0,021$. Respecto del análisis de comparación de medias utilizando ANOVA de medidas repetidas entre los momentos 2 y 3 sobre los datos del cuestionario utilizado, comprobamos que no se registran cambios significativos entre los momentos 2 y 3 , salvo para el ítem 10 ("Deberían existir deportes específicos exclusivos para cada discapacidad") en el cual se observa como disminuye la media trascurridos 9 meses, produciéndose un cambio significativo en positivo. 
Pérez-Tejero, J.; Ocete-Calvo, C.; Ortega-Vila, G.; Coterón-López, J. (2012). Diseño y aplicación de un programa de intervención de práctica deportiva inclusiva y su efecto sobre la actitud hacia la discapacidad: El Campus Inclusivo de Baloncesto. RICYDE. Rev. int. cienc. deporte. 29(8), 258-271. http://dx.doi.org/10.5232/ricyde2012.02905

Tabla 4. Comparación momento 2 y 3. *: Diferencia significativa a $\mathrm{p}<0,05$.

\begin{tabular}{|c|c|c|c|c|c|c|c|}
\hline Ítem & $\begin{array}{c}\text { Dif. medias } \\
(2-3)\end{array}$ & $\begin{array}{l}\text { Error } \\
\text { típico }\end{array}$ & Sig. ${ }^{A}$ & Ítem & $\begin{array}{l}\text { Dif. medias } \\
(2-3)\end{array}$ & $\begin{array}{l}\text { Error } \\
\text { típico }\end{array}$ & Sig. ${ }^{A}$ \\
\hline 1 & $-1,154$ & ,597 &, 077 & 10 &, $769 *$ & ,343 &, 044 \\
\hline 2 & $-1,154$ & ,597 & ,077 & 11 & ,000 & ,000 & . \\
\hline 3 &, 615 &, 656 &, 367 & 12 & 083 & ,193 &, 674 \\
\hline 4 &,- 308 & ,365 & ,416 & 13 & 0,77 & 0,77 & ,337 \\
\hline 5 & ,462 & ,462 & ,337 & 14 &,- 615 & ,401 & ,151 \\
\hline 6 &,- 091 & ,211 & 676 & 15 & 0,77 & ,431 &, 861 \\
\hline 7 & 615 & ,290 & 055 & 16 & .385 & ,368 &, 316 \\
\hline 8 &,- 615 & ,331 &, 088 & 17 &,- 154 &, 191 &, 436 \\
\hline 9 & ,000 & ,113 & 1,00 & & & & \\
\hline
\end{tabular}

\section{Discusión}

El objetivo del estudio fue diseñar una propuesta de intervención como modelo de práctica deportiva inclusiva. A nivel nacional, este tipo de intervención en el ámbito del ocio y recreación es pionera por su metodología inclusiva. El acceso a una muestra siginificativa de personas con y sin discapacidad coincidiendo en una practica deportiva inclusiva no es habitual en este ámbito. Por este motivo la mayoría de los estudios planteados se producen en un contexto educativo, al tener un acceso más fácil a la muestra debido a su carácter de obligatoriedad legislativa. Sin embargo, lo determinante en estos casos es la interacción producida entre personas con y sin discapacidad, condicionando las intervenciones y quedando en un segundo plano los entornos en los que se produce.

Se debe hacer una distinción entre los estudios planteados desde una perspectiva integradora respecto a los estudios enfocados desde una perspectiva inclusiva como es este caso. La metodología inclusiva, favorece el proceso social de inclusión normalizándolo de cara a un futuro, como nivel superior a la metodología integradora, en nuestra opinión. Reseñado esto, uno de los puntos clave en el diseño de esta intervención ha sido la selección combinada de las técnicas de información y CD. El estudio coincide con otros similares en el ámbito educativo: Aguado, Alcedo, y Arias (2008) en una intervención con alumnos de educación primaria utilizaron ambas técnicas resultando efectivas para el cambio, aunque en su intervención el contacto se produjo de manera intermitente y no especificamente en el ámbito de la Educación Física. Suriá (2011) en un estudio comparativo de estudiantes de secundaria y universitarios, utilizó el CD para ambos grupos pero tampoco se produjo en un contexto deportivo. De la misma manera que para Pernice y Lys (1996), que con estudiantes universitarios obtuvieron resultados positivos tras el CD con personas con discapacidad en un entorno académico.

Sin embargo, este CD no tendría sentido si no se produjese de manera frecuente ya que, como afirman Jacques y col. (1998), cuanto más tiempo de CD se produzca más posibilidad de actitudes positivas tendremos. Reina y col. (2011) demuestran cómo una intervención de un programa de 6 días, causa un efecto superior en la actitud frente a uno de un solo día. En nuestra intervención de 4 días de duración, no solo facilitamos el 
Pérez-Tejero, J.; Ocete-Calvo, C.; Ortega-Vila, G.; Coterón-López, J. (2012). Diseño y aplicación de un programa de intervención de práctica deportiva inclusiva y su efecto sobre la actitud hacia la

discapacidad: El Campus Inclusivo de Baloncesto. RICYDE. Rev. int. cienc. deporte. 29(8), 258-271. http://dx.doi.org/10.5232/ricyde2012.02905

contacto en el ámbito recreativo, sino también que los participantes tuviesen contacto en el ámbito personal, recibiendo información directa e indirecta sobre la discapacidad y rompiendo así los estereotipos negativos sobre ellos. La duración del contacto es determinante, pero no está claro en la literatura que su distribución en el tiempo lo sea, ya que hay disparidad en los autores consultados en función de los planteamientos de intervención. Así, Aguado, Alcedo y Arias (2008) y Tripp, French y Sherrill (1995) dividieron el contacto en diferentes sesiones, Bergman y Hanson (2000) durante días de manera continuada. El CD entre personas sin discapacidad en un entorno de ocio y recreación está normalizado, no siendo el tiempo una variable limitadora a tener en cuenta en el desarrollo de programas y actividades. En el caso de los programas inclusivos, conseguir un CD prolongado es más costoso por los requisitos indispensables de acceso para las personas con discapacidad.

El apoyo institucional permitió disponer de unas instalaciones y unos recursos materiales adecuados para la práctica, facilitando así una participación activa por parte de los jugadores con discapacidad. Consideramos este aspecto de gran relevancia ya que posibilita centrar la atención en las habilidades necesarias para la práctica deportiva en lugar de las limitaciones propias de la discapacidad. Al igual que Block y Zeman (1996), estamos de acuerdo en que los chicos con discapacidad se pueden incluir en Educación física con sus compañeros que no la tienen, sin que ello afecte negativamente al programa, pero siempre y cuando se les facilite los apoyos y recursos necesarios para que participen de manera activa en las sesiones. Disponer de recursos materiales adaptados y de unas instalaciones accesibles es una condición indispensable para el planteamiento y desarrollo de programas deportivos inclusivos. No solo se debe recurrir al apoyo institucional en casos puntuales, sino que se deben promover políticas inclusivas reales y activas donde se atienda a la demanda social. Sirviéndonos de una accesibilidad universal como base de actuación en todos los ámbitos, promoveremos de esta manera la normalización en el tratamiento de la discapacidad.

Un aspecto a destacar en el diseño de la intervención fue el papel desempeñado por los técnicos responsables de la actividad. Hodge, Davis, y col. (2002) en un estudio realizado para la formación de dos grupos de profesores de Educación Física, concluyeron la importancia que tiene la formación específica para la mejora de las actitudes. En nuestro caso, todos los profesionales a cargo de la intervención poseían titulaciones específicas en baloncesto, Ciencias de la Actividad Física y del Deporte e inclusión educativa, así como más de 5 años de experiencia previa en estos contextos. Esto permitió realizar las adaptaciones pertinentes de manera planificada previamente al desarrollo del campus, desarrollando actividades y tareas concretas donde las situaciones inclusivas cada vez iban adquiriendo mayor protagonismo. Además, la formación específica en la materia permite a los técnicos modificar in situ las cuestiones que van surgiendo durante la práctica, facilitando la participación activa por parte de los jugadores con discapacidad en cada momento y resolviendo los conflictos propios de la práctica. Esta fue una de las claves del éxito de esta intervención ya que facilitó la creación de un contexto adecuado para que se produjese la interacción entre personas con y sin discapacidad.

Medir el impacto de dicha intervención en la actitud hacia la discapacidad era el segundo objetivo planteado en este estudio. A la luz de los resultados obtenidos, se encuentra una tendencia general hacia una actitud más positiva tras la intervención, mostrando los participantes una mejor aceptación de la discapacidad, coincidiendo así 
Pérez-Tejero, J.; Ocete-Calvo, C.; Ortega-Vila, G.; Coterón-López, J. (2012). Diseño y aplicación de un programa de intervención de práctica deportiva inclusiva y su efecto sobre la actitud hacia la

discapacidad: El Campus Inclusivo de Baloncesto. RICYDE. Rev. int. cienc. deporte. 29(8), 258-271. http://dx.doi.org/10.5232/ricyde2012.02905

con los resultados de Slininger y col. (2000); Hodge, Davis y col. (2002); Block y Malloy (1998); Aguado y col. (2008). Diferimos de autores como Panagiotou, Evaggelinou, Doulkeridou, Koidou y Mouratidou (2009); Liu y col. (2010), Jesina y col. (2006), o Van Biesen, Busciglio y Vanlandewijck (2006), ya que en este estudio los jugadores han mostrado una actitud favorable tras la adaptación y modificación de las reglas del juego. Esto puede ser debido al conjunto de todas las variables mencionadas anteriormente que han fundamentado el planteamiento metodológico del campus y que, creemos, han facilitado el desarrollo de una intervención inclusiva de calidad.

Estos resultados coinciden con los obtenidos por Block y Zeman (1996) y Gillespie (2002), ambos en campus recreativos similares al presentado en este estudio. Pese a los resultados positivos obtenidos tras la intervención, se encontró menor disposición a participar en actividades competitivas de carácter inclusivo. Esto puede deberse a que la competición puede ser percibida como una barrera en la inclusión a través de la práctica deportiva (Block, 1995), ya que puede tomar una dirección negativa al no proporcionar igualdad de estatus en los participantes y no poseer un objetivo común. La puesta en práctica de las variables anteriormente mencionadas nos reportó los resultados positivos obtenidos tras el estudio.

Al estudiar si dichos cambios se mantenían en el tiempo, tras 9 meses finalizada la intervención, se comprobó una consistencia significativa. Coincidimos con los estudios de Aguado y col. (2008) y de Krahé y Altwasser (2006) donde los cambios perduraron en el tiempo. Este punto es clave en esta intervención ya que supone un importante apoyo que refuerza el impacto de este tipo de diseños. Por regla general, los estudios planteados miden la actitud antes y después de una intervención, no comprobando si dichos cambios se mantienen en el tiempo y esto es un aspecto fundamental a la hora de implantar intervenciones de este tipo. Para futuros estudios sería interesante medir la existencia de variables que influyen en el mantenimiento de este cambio.

Una de las limitaciones habituales en los estudios relacionados con actividad física adaptada es la dificultad de acceso a la muestra por el reducido tamaño de la población y el escaso porcentaje de la misma que incluye actividad física en su vida cotidiana. El que la intervención propuesta tuviese carácter inclusivo condicionaba todavía más la selección de los participantes; pese a ello, consideramos satisfactorias las características del estudio. Otra limitación fue la mortalidad de parte de la muestra en el tercer momento del estudio, aunque en este tipo de estudios longitudinales suele ser habitual. Por último, cabe reseñar que las características del estudio son difíciles de reproducir tal y como se ha planteado en esta investigación, ya que en el ámbito del deporte para personas con discapacidad habitualmente se encuentran limitaciones tales como poca implicación a nivel institucional, espacios poco accesibles, material adaptado de elevado coste económico y falta de profesionales cualificados. Para promover este tipo de estudios y aumentar la producción científica que permita, a medio plazo, la elaboración de modelos teóricos válidos, se propone extrapolar este diseño de intervención a contextos reales de práctica, dejando de ser una actividad de carácter puntual para convertirse en una práctica habitual y continuada de deporte inclusivo.

Si nos sirviésemos de este tipo de propuestas en todos los ámbitos y niveles de actuación, desde las edades tempranas, donde las actitudes aún no están consolidadas (Nowicki, 2006), creemos que no sería tan necesario el paliar las actitudes negativas, ya que desde esas edades se comenzaría el proceso de socialización e interacción con el mundo que les rodea de manera adecuada y normalizada, en beneficio del fomento de 
las actitudes no discriminatorias hacia los iguales con discapacidad. Esto nos lleva a abordar el futuro con un doble planteamiento: por un lado, ofertar actividades de forma continua y de calidad que fomenten la inclusión; por otro, crear un modelo de intervención fundamentado en la teoría y en los datos de la investigación (Hutzler, 2003), que permita elaborar una estructura de trabajo transferible a otros entornos de enseñanza para el desarrollo teórico-práctico de modelos de intervención inclusiva. Nuestro modelo propuesto intenta arrojar luz en esa línea, demostrando sus efectos beneficiosos al acabar la intervención y tras un lapso temporal significativo.

\section{Conclusiones}

El modelo de intervención propuesto de práctica deportiva inclusiva parece ser un diseño completo y eficaz para la modificación en positivo de las actitudes hacia las personas con discapacidad. Por otro lado, se demuestra el impacto favorable del Campus Inclusivo de Baloncesto sobre la actitud de los participantes sin discapacidad, permaneciendo dichos cambios en el tiempo. Queda patente la necesidad de una coordinación que facilite la inclusión de las personas con discapacidad en la práctica, no solo por parte de los profesionales del ámbito deportivo, sino también a nivel institucional, basada en la formación específica de los técnicos y el acceso universal en espacios y materiales. De esta manera, el acceso a la práctica deportiva se convierte en una herramienta normalizadora y de inclusión social de primer orden.

\section{Bibliografía}

Aguado, A.; Alcedo, M., y Arias, B. (2008). Cambio de actitudes hacia la discapacidad con escolares de primaria. Phicothema, 20(4), 697-704.

Ajzen, I., \& Fishbein, M. (Eds.). (1980). Understanding attitudes and predicting social behavior. New Jersey: Prentice Hall, Inc.

Allport, G. (Ed.). (1954). The nature of prejudice. New York: Doubleday Books.

Antonak, R., \& Livneh, H. (Eds.). (1988). The measurement of attitud toward people with disabilities. Springfield: Charles C.Thomas.

Archie, V., \& Sherrill, C. (1989). Attitudes toward handicapped peers of mainstreamed and nonmainstreamed children in physical education. Perceptual Motor Skills, 69(1), 319-322.

Bergman, M., \& Hanson, C. (2000). The relationship between participation in a sports camp and students' attitudes towards persons with disabilities. Occupational Therapy in Health Care, 12(4), 51-63.

Blinde, E., \& McClung, L. (1997). Enhancing the Physical and social self through recreational activity: accounts of individuals with Physical disabilities. Adapted Physical Activity Quarterly, 14(4), 327-344.

Block, M. (1995). Development and Validation of the Children's Attitudes Toward Integrated Physical Education - Revised (CAIPE-R) Inventory. Adapted Physical Activity Quarterly, 12(1), 60-77.

Block, M., \& Malloy, M. (1998). Attitudes on inclusion of a player with disabilites in a regular softball league. Mental Retardation, 36(2), 137-144. 
Pérez-Tejero, J.; Ocete-Calvo, C.; Ortega-Vila, G.; Coterón-López, J. (2012). Diseño y aplicación de un programa de intervención de práctica deportiva inclusiva y su efecto sobre la actitud hacia la

discapacidad: El Campus Inclusivo de Baloncesto. RICYDE. Rev. int. cienc. deporte. 29(8), 258-271. http://dx.doi.org/10.5232/ricyde2012.02905

Block, M., \& Zeman, R. (1996). Including students with disabilities in regular physical education: effects on nondisabled children. Adapted Physical Activity Quarterly, 13(1), 38-49.Brasile, F. (1990). Wheelchair sports: a new perspective on integration. Adapted Physical Activity Quarterly, 7(1), 3-11.

Conaster, P.; Block, M. E., \& Gansneder, B. (2002). Aquatic instructors' beliefs toward inclusion: the theory of planned behavior. Adapted physical activity quarterly, 19(2), 172-187.

Finkelsteins, V., \& French, S. (1993). Towards a psychology of disability. En Sage (Ed.), Disabling barriers: Enabling environments. (pp. 26-33). London.

Flórez, M.; Aguado, A., y Alcedo, M. (2009). Revisión y análisis de los programas de cambio de actitudes hacia personas con discapacidad. Anuario de Psicología clínica y de la salud., 5, 85-98.

Folsom-Meek, S., \& Rizzo, T. (2002). Validating the Physical Educators' Attitude Toward Teaching Individuals With Disabilities III (PEATID III) survey for future professionals. Adapted Physical Activity Quarterly, 19(2), 141.

Gillespie, M. (2002). Attitudes of university students toward an integrated campus recreation program. Palaestra, 18(3), 27-31.

Heikinaro-Johansson, P.; French, R.; Sherrill, C., \& Huuhka, H. (1995). Adapted physical education consultant service model to facilitate integration. Adapted Physical Activity Quarterly, 12(1), 12-33.

Hodge, S.; Davis, R.; Woodard, R., \& Sherrill, C. (2002). Comparison of practicum types in changing preservice teachers' attitudes and perceived competence. Adapted Physical Activity Quarterly, 19(2), 155-171.

Hodge, S.; Murata, N., \& Kozub, F. (2002). Physical educators' judgments about inclusion: a new instrument for preservice teachers. Adapted Physical Activity Quarterly, 19(4), 435-452.

Hutzler, Y. (2003). Attitudes toward the participation of individuals with disabilities in physical activity: a review. Quest, 55(4), 347-373.

Jacques, N.; Wilton, K., \& Townsend, M. (1998). Cooperative learning and social acceptance of children with mild intellectual disability. Journal of Intellectual Disability Research, 42(1), 29-36.

Jesina, O.; Lucas, S.; Kudlacek, M.; Machova, I.; Janecka, Z., \& Wittmannova, J. (2006). Effect of an intervention program on attitude of elementary school children toward inclusion of children with a disability. Documento presentado en el 8th European Congress of Adapted Physical Activity, Olomouc, Rep.Checa.

Kowalski, E., \& Rizzo, T. (1996). Factors influencing preservice student attitudes toward individuals with disabilities. Adapted Physical Activity Quarterly, 13(2), 180-196.

Krahé, B., \& Altwasser, C. (2006). Changing negative attitudes towards persons with physical disabilities: an experimental intervention. Journal of Community and Applied Social Psychology, 16(1), 59-69.

Liu, Y.; Kudlacek, M., \& Ondrej, J. (2010). The influence of paralympic choool day on children's attitudes towards people with disabilities. Acta Universitatis Palackianae Olomucensis. Gymnica 40(2), 63-69.

Nowicki, E. (2006). A cross-sectional multivariate analysis of children's attitudes towards disabilities. J ournal of Intellectual Disability Research, 50(5), 335-348. 
Panagiotou, A.; Evaggelinou, C.; Doulkeridou, A.; Koidou, E., \& Mouratidou, K.(2009). Evaluation of student's attitudes from conventional primary schools toward inclusion of children with disabilities in physical education after the implementation of a program. Inquiries in Sport and Physical Education, 7(2), 103-113.

Pérez, J.; Ortega, G.; Trigo, O., y Pinilla, J. (2009). I Campus Inclusivo de Baloncesto de la Fundación Real Madrid. I J ornadas de Educación Física, inclusión y alumnado con discapacidad. Barcelona: UB e Instituto de Ciencias de la Educación.

Pernice, R., \& Lys, K. (1996). Interventions for attitude change towards people with disabilities: how successful are they? International Journal of Rehabilitation Research, 19(2), 171-174.

Reina, R. (2003). Propuesta de intervención para la mejora de las actitudes hacia las personas con discapacidad a través de actividades deportivas y recreativas Acceso el 19 Abril 2011, recuperado de http://www.efdeportes.com/efd59/discap.htm

Reina, R.; Jiménez, M.; López, V., y Menayo, R. (2008). Mejora las actitudes hacia las personas con discapacidad en escolares tras participar en una unidad didáctica de sensibilización de fútbol sala para ciegos. León: $V$ congreso en la asociación española de ciencias del deporte.

Reina, R.; López, V.; Jiménez, M.; García-Calvo, T., \& Hutzler, Y. (2011). Effects of awareness interventions on children's attitudes toward peers with a visual impairment. International J ournal of Rehabilitation Research, 34 (3), 243-248.

Ríos, M.; Arraez, J.; Bazalo, P.; Enciso, M.; Hueli, J.; Jiménez, E., y col. (2009). Plan integral para la Actividad Física y el Deporte. Versión 1.No definitiva. Acceso el 12 de Diciembre de 2011, recuperado de http://www.csd.gob.es/csd/estaticos/plan-integral/discapacidad.pdf

Ruiz, L. (Ed.). (2002). Animación y discapacidad. La integración en el tiempo libre. Salamanca: Amarú.

Shaver, J. (1987). The modification of attitudes toward persons with handicaps: a comprehensive integrative review of research. Final Report. Utah State Univ, Logan Bureau of Educational Research.

Sherrill, C. (2003). Should social inclusion be a major goal of physical education? Palaestra, 19(2), 56-57.

Sherrill, C. (Ed.). (1998). Adapted physical activity, recreation and sport: crossdisciplinary and lifespan (5th ed.). Dubuke: Mcgraw-Hill.

Siperstein, G.; Norins, J.; Corbin, S., \& Shirver, T. (2003). Estudio multinacional de actitudes hacia las personas con discapacidad. Acceso el 1 de noviembre de 2011, recuperado de www.specialolympics.org

Slininger, D.; Sherrill, C., \& Jankowski, C. (2000). Children's attitudes toward peers with severe disabilities: revisiting contact theory. Adapted Physical Activity Quarterly, 17(2), 176-196.

Suriá, R. (2011). Análisis comparativo sobre las actitudes de los estudiantes hacia sus compañeros con discapacidad. Revista Electrónica de Investigación Psicoeducativa y Psicopedagógica. Acceso el 10 de noviembre de 2011, recuperado de http://www.investigacion-

psicopedagogica.org/revista/new/ContadorArticulo.php?511 
Tripp, A.; French, R., \& Sherrill, C. (1995). Contact theory and attitudes of children in physical education programs toward peers with disabilities. Adapted physical activity quarterly, 12(4), 323-332.

Van Biesen, D.; Busciglio, A., \& Vanlandewijck, Y. (2006). Attitudes towards inclusion of children with disabilities: the effect of the implementation of "A Paralympic School Day" on Flemish elementary children. 8th European Conference of Adapted Physical Activity Acceso el 26 octubre de 2011, recuperado http://www.eufapa.upol.cz/index.php/eucapa/proceedings/eucapa-2006.html

Verdugo, M.; Jenaro, C., y Arias, B. (Eds.). (1994). Actitudes hacia las personas con minusvalía. Madrid: IMSERSO.

Wilhite, B.; Mushett, C.; Goldenberg, L., \& Trader, B. (1997). Promoting inclusive sport and leisure participation: Evaluation of the Paralympic Day in the schools model. Adapted Physical Activity Quarterly, 14(2), 131-146.

Williams, T. (1994). Disability sport socialization and identity construction. Adapted Physical Activity Quarterly, 11(1), 14-31.

Xafopoulos, G.; Kudlacek, M., \& Evaggelinou, C. (2009). Effect of the intervention program "Paralympic school day" on attitudes of children attending international school towards inclusion of students with disabilities. Documento presentado en el Acta Universitatis Palackianae Olomucensis. Gymnica. 